\title{
Weight change and glycemic control in type 2 diabetes patients during COVID-19 pandemic: the lockdown effect
}

\author{
Emilia Biamonte $^{1,2} \cdot$ Francesco Pegoraro $^{1,2} \cdot$ Flaminia Carrone $^{1,2} \cdot$ Isabella Facchi $^{1,2} \cdot$ Giuseppe Favacchio $^{1}$. \\ Andrea Gerardo Lania ${ }^{1,2} \cdot$ Gherardo Mazziotti $^{1,2} \cdot$ Marco Mirani $^{1}$
}

Received: 17 December 2020 / Accepted: 21 April 2021 / Published online: 5 May 2021

(c) The Author(s), under exclusive licence to Springer Science+Business Media, LLC, part of Springer Nature 2021

\begin{abstract}
Purpose The aim of this study was to evaluate the impact of the COVID-19 lockdown occurred in Italy from March 9th to May 18th, 2020 on anthropometric parameters and glycemic control in patients with type 2 diabetes mellitus (T2DM). Methods One hundred twenty-eight consecutive patients with T2DM (median age 70 years, 74 males) were retrospectively evaluated at the end of the lockdown period. Data on metabolic control were collected at different time: within three months before the lockdown (visit 0 ) and within the first six weeks after it (visit 1).

Results During the lockdown, a significant increase in body weight (from $79.7 \pm 18.7 \mathrm{~kg}$ to $81.4 \pm 19.4 \mathrm{~kg}, p<0.001$ ), body mass index (BMI, from $29.5 \pm 6 \mathrm{~kg} / \mathrm{m}^{2}$ to $30.1 \pm 6.3 \mathrm{~kg} / \mathrm{m}^{2}, p<0.001$ ), waist circumference (from $103.8 \pm 13 \mathrm{~cm}$ to $105 \pm$ $13.6 \mathrm{~cm}, p<0.001$ ), fasting plasma glucose (FPG; from $138.1 \pm 29.4 \mathrm{mg} / \mathrm{dL}$ to $146.6 \pm 36.4 \mathrm{mg} / \mathrm{dL}$ ) and glycated hemoglobin (HbA1c; from $7 \pm 0.8$ to $7.3 \pm 0.9 \%, p<0.001$ ) was observed. Weight gain was directly associated with HbA1c increase $(\beta 0.085$, C.I. $95 \% 0.05-0.121 ; p<0.001)$ while insulin therapy resulted to be the only significant independent predictor of HbA1c worsening at the multivariate logistic regression analysis (OR 2.40, C.I. 1.06-5.45; $p=0.035$ ).

Conclusions The lockdown due to COVID-19 pandemic had a negative impact on body weight and glucose control in T2DM patients, in particular in those on insulin treatment. This finding provides a further rationale to optimize the diabetes management during eventually new period of home confinement.
\end{abstract}

Keywords COVID-19 $\cdot$ Type 2 diabetes mellitus $\cdot$ Weight change $\cdot$ Glycemic control-lockdown

\section{Introduction}

The outbreak of the COVID-19 pandemic has brought a worldwide threat to public health. To contain the spread of the virus, from March 9th to May 18th, 2020 Italian government imposed a nationwide lockdown. Millions of people were confined indoors to drastically minimize any human contact, and non-essential working activities were stopped or switched to home working. In the health facilities, all outpatient activities were discontinued, as most of

Marco Mirani

marco.mirani@humanitas.it

1 Endocrinology, Diabetology and Andrology Unit, IRCCS Humanitas Research Hospital, Rozzano (MI), Italy

2 Department of Biomedical Sciences, Humanitas University, Pieve Emanuele (MI), Italy the healthcare professionals were assigned to COVID19 wards.

These necessary social distancing policies and public health re-organization have understandably introduced many challenges in the management of chronic diseases [1]. Among them, satisfactory control of type 2 diabetes mellitus (T2DM) has several pitfalls, due to sub-optimal medical surveillance and to an abrupt change in the patient's lifestyle [2, 3].

Previous reports have already shown that dietary habits of most Italian families changed during the lockdown, due both to more free-time availability and to variation of food expenditure. Time spent cooking increased, as well as the nourishing with high glycemic index foods, like homemade pizza, pasta, bread, and desserts. Furthermore, the home confinement reduced the opportunities for physical exercise [4-6].

According to ADA and EASD guidelines, healthy habits are the foundations of the clinical management of T2DM $[7,8]$. Reduced physical activity and increased body weight 
have a detrimental effect on glycemic control with a consequent higher risk of diabetes complications [9].

To our acknowledge, there still is a paucity of real-world data on the impact of lockdown measures on body composition and glycemic control in T2DM [6, 10-14]. As a matter of fact, the Italian lockdown is an unprecedented and hopefully unique occasion to study the effect of lifestyle changes on T2DM control in the real life.

Giving these premises, in this study we aim to evaluate the changes of anthropometric parameters and glycemic control in a homogeneous population of T2DM.

\section{Material and methods}

\section{Study design}

This is a retrospective observational study based on the medical records of 128 consecutive subjects with T2DM. The outpatients were evaluated at the Diabetology Unit of Humanitas Clinical and Research Center, IRCCS (Rozzano, Milan - Italy) at baseline (visit 0), between December 15th, 2019 and the March 1st, 2020 and at the resumption of clinical activities (visit 1), between May 15th and June 30th, 2020. None of the subjects was diagnosed with SARS-CoV2 infection. The lockdown period occurred between March 14th and April 30th, 2020.

The inclusion criteria were: 1 ) age $\geq 18$ years; 2 ) history of T2DM for more than 6 months prior to the lockdown; 3 ) full availability of anti-diabetic medication history, anthropometric parameters, glycemia and $\mathrm{HbA} 1 \mathrm{c}$ during the preand the immediate post-lockdown period; 4) no weight change of $\pm 1.5 \mathrm{~kg}$ and no $\mathrm{HbA} 1 \mathrm{c}$ variation of $\pm 0.3 \%$ between the visit 0 and the previously available visit (not less than six months before the lockdown period). The exclusion criteria were: 1) change in antidiabetic therapy at least 6 months before the lockdown; 2) onset of illness and history of hospitalization potentially influencing diabetes control in the 3 months prior to the lockdown and during it, including SARS-CoV-2 infectious; 3) state of pregnancy; 4) active oncological disease.

The primary endpoint of this study was to evaluate the impact of the lockdown-effect on patients' anthropometric parameters and glycemic status. As secondary endpoints, we explored the determinants of worsened glycaemic control.

Clinical information and outpatients' reports were retrospectively acquired, using the available clinical files. Gender, age, duration of diabetes, diabetes-related complications, co-morbidities, weight, body mass index (BMI), waist circumference, detailed anti-diabetic therapy, glycated hemoglobin (HbA1c), and fasting plasma glucose (FPG) were collected for each patient. Data on glucose control and on anthropometrics parameters were collected at different times: within three months before the lockdown (visit 0) and within the first six weeks after the quarantine (visit 1). The glycemic control changes were evaluated as variations of HbA1c and FPG between visit 0 and visit 1. The same method was applied to define the anthropometric changes.

The study was approved by the Ethical Committee of Humanitas Clinical and Research Center, IRCCS, Rozzano - Milan, and patients gave their consent to use clinical data for research purposes.

\section{Statistical analysis}

Continuous variables were reported as median and range, unless otherwise stated. The biochemical and anthropometric parameters evaluated before and after the lockdown were compared using the Wilcoxon Signed Rank Test. Statistical significance was assumed when $P \leq 0.05$. Univariate linear regression analysis was performed to define the associations between $\mathrm{HbA} 1 \mathrm{c}$ and continuous variables. To explore the predictive factor associated with $\mathrm{HbA1c}$ worsening an univariate logistic regression analysis was performed and the OR, and $95 \%$ confidence intervals $(95 \%$ CI) were calculated. All factors with a $P$ value under 0.100 were then submitted to a multivariable logistic regression analysis.

The multicollinearity test between variables included in the multivariate analysis was performed: variable with a tolerance $(\mathrm{T})<0.2$ and a variance inflation factor $(\mathrm{VIF})>10$ was excluded.

\section{Results}

At the resumption of outpatients' activities after the lockdown period (between May 15th and June 30th, 2020), a total of 485 diabetic patients were evaluated in our Institution. Among them, 128 patients (54 females, 74 males) fulfilled the inclusion criteria and were enrolled in the study. The median age of the study population was 70 years (range: 40-91). The median diabetes disease duration was 15 years (range: $2-50$ ).

Fifteen patients $(11.7 \%)$ were on monotherapy, 7 (5.5\%) of which with metformin, whereas $113(88.3 \%)$ were taking two or more antidiabetic drugs. In the multi-therapy group, 97 patients $(75.8 \%$ of the total population) were taking metformin plus another anti-diabetic drug. Forty-two $(32.3 \%)$ patients were treated with GLP-1 receptor agonist, 33 (25.4\%) with SGLT2 inhibitors, 32 (24.6\%) with DPP4 inhibitors, $24(18.5 \%)$ with sulfonylureas, and 42 $(32.3 \%)$ with insulin therapy. Details on hypoglycemic treatment and on clinical data at the baseline are shown in the Table 1. 
Table 1 Clinical data and antidiabetic treatment of study population at baseline (visit 0)

\begin{tabular}{|c|c|c|}
\hline Variable & Patients N. $($ Total $=128)$ & Value- $\%$ \\
\hline \multicolumn{3}{|l|}{ Clinical parameters } \\
\hline Male & 74 & 57.8 \\
\hline Age (median; range) & $70(40-91)$ & - \\
\hline Smoker & 42 & 32 \\
\hline Hypertension & 105 & 82 \\
\hline Dyslipidemia & 88 & 68.8 \\
\hline CAD & 32 & 25 \\
\hline Heart failure & 25 & 19.5 \\
\hline Stroke & 7 & 5.5 \\
\hline CKD $(\mathrm{eGRF}<60 \mathrm{ml} / \mathrm{min})$ & 46 & 35.9 \\
\hline Microalbuminuria (>30 mg/l) & 23 & 18 \\
\hline Neuropathy & 17 & 13.3 \\
\hline Retinopathy & 33 & 25.8 \\
\hline \multicolumn{3}{|l|}{ Antidiabetic treatment } \\
\hline Monotherapy & 15 & 11.7 \\
\hline Multi-therapy $^{\mathrm{a}}$ & 113 & 88.3 \\
\hline Metformin & 104 & 81.3 \\
\hline SU & 24 & 18.5 \\
\hline Insulin & 42 & 32.3 \\
\hline SGLT2-I & 33 & 25.4 \\
\hline DPP4-I & 32 & 24.6 \\
\hline GLP-1 RA & 42 & 32.3 \\
\hline Pioglitazone & 14 & 10.8 \\
\hline
\end{tabular}

$C A D$ coronary artery disease, $C K D$ chronic kidney disease, $e G F R$ estimated Glomerular Filtration Rate, $S U$ sulfonylureas, SGLT2i sodium glucose cotransporter 2 inhibitors, $D P P 4 i$ dipeptidyl peptidase IV inhibitors, GLP-1 RA glucagon-like peptide-1 receptor agonists

${ }^{a} 97$ out of 113 patients on anti-diabetic polytherapy were in metformin in addition of another class-drug

Table 2 Clinical and biochemical evaluation before the lockdown (visit 0) and in the immediate post-lockdown period (visit 1)

\begin{tabular}{lccc}
\hline Variable & Visit 0 & Visit 1 & $P$ value \\
& $N=128$ & $N=128$ & \\
\hline Anthropometric parameters & & & \\
Weight $(\mathrm{kg})$ & $79.7 \pm 18.7$ & $81.4 \pm 19.4$ & $<0.001$ \\
Waist circumference $(\mathrm{cm})$ & $103.8 \pm 13$ & $105 \pm 13.6$ & $<0.001$ \\
BMI $\left(\mathrm{kg} / \mathrm{m}^{2}\right)$ & $29.5 \pm 6$ & $30.1 \pm 6.3$ & $<0.001$ \\
Biochemical parameters & & & \\
FPG $(\mathrm{mg} / \mathrm{dL})$ & $138.1 \pm 29.4$ & $146.6 \pm 36.4$ & $<0.001$ \\
HbA1c \% & $7 \pm 0.8$ & $7.3 \pm 0.9$ & $<0.001$ \\
HbA1c $(\mathrm{mmol} / \mathrm{mol})$ & $53 \pm 9$ & $56 \pm 10$ & $<0.001$ \\
\hline
\end{tabular}

$B M I$ body mass index, $F P G$ fasting plasma glucose, $H b A l c$ glycated hemoglobin

Anthropometric and biochemical data at baseline (visit 0) and immediately after the end of the lockdown (visit 1) are reported in Table 2. During the observational period, all the evaluated anthropometric parameters were significantly increased (body weight from $79.7 \pm 18.7 \mathrm{~kg}$ to $81.4 \pm$ $19.4 \mathrm{~kg}, p<0.001$; BMI from $29.5 \pm 6 \mathrm{~kg} / \mathrm{m}^{2}$ to $30.1 \pm$ $6.3 \mathrm{~kg} / \mathrm{m}^{2}, p<0.001$; waist circumference from $103.8 \pm$ $13 \mathrm{~cm}$ to $105 \pm 13.6 \mathrm{~cm}, p<0.001)$. FPG and $\mathrm{HbA} 1 \mathrm{c}$ were significantly worsened (FPG from $138.1 \pm 29.4 \mathrm{mg} / \mathrm{dL}$ to $146.6 \pm 36.4 \mathrm{mg} / \mathrm{dL}$ and $\mathrm{HbA} 1 \mathrm{c}$ from $7 \pm 0.8$ to $7.3 \pm 0.9 \%$, $p<0.001)$.

At the univariate linear regression analysis, weight, BMI and WC changes were directly correlated with $\mathrm{HbA1c}$ variations (weight: $\beta 0.085$, C.I. 95\% $0.05-0.121 ; p<$ 0.001; BMI: $\beta$ 0.246, C.I. 95\% 0.15-0.343; <0.001; WC: $\beta$ 0.068 , C.I. $95 \% 0.032-0.104 ;<0.001)$. Given the collinearity of these variables, only the estimated association curve between weight changes and HbAlc variation is reported in Fig. 1.

In the univariate logistic regression, insulin therapy (OR 2.45 , C.I. $95 \% 1.09-5.05, p=0.030$ ), the presence of CAD (OR 2.43, C.I. 95\% 0.99-5.96; $p=0.052$ ) and the presence of chronic kidney disease (CKD) (OR 2.37, C.I. 1.07-5.23; $p=0.032$ ) were significant predictors of $\mathrm{HbA} 1 \mathrm{c}$ worsening. In the multivariate model, having excluded the CKD because of its strong association with insulin use (47.6\% of patients with CKD were on insulin therapy), insulin therapy remains the only independent significant predictor of HbA1c increase (OR 2.40, C.I. 1.06-5.45; $p=0.035$ ) Table 3. We then compared the clinical characteristics of insulin and non-insulin-treated groups - Table 4. The T2DM duration, the basal $\mathrm{HbAlc}$ value, the presence of heart failure and retinopathy were higher in the insulin-treated group; instead, the concomitant use of metformin, DPP4-I and pioglitazone were significantly higher in non-insulintreated patients.

\section{Discussion}

This monocenter study evaluated the impact of lockdown on anthropometric parameters and glycemic control in a population of adult patients with T2DM. Most of the previous papers on glycemic control during the COVID-19 pandemic were focused on type 1 diabetes mellitus (T1DM) patients, especially those using novel glucose-sensing technology [10, 12, 14-16].

In our study, the homogeneity of our cohort, the inclusion of only patients with stable weight and glucose control, and the lack of medical contact (both in-person and virtually) allowed us to use the quarantine as a model to better define its effect on T2DM control and body weight.

The importance of satisfactory glycemic and weight control in outpatient diabetic subjects is an emergent hot topic in the COVID-19 era. Several recent findings have suggested that, when infected, an inadequate metabolic 
Fig. 1 Linear correlation between weight gain and $\mathrm{HbAlc}$ variation. $R^{2} 0.152(p<0.001)$
Table 3 Results of univariate and multivariate logistic regression analyses evaluating the determinants of $\mathrm{HbA} 1 \mathrm{c}$ worsening

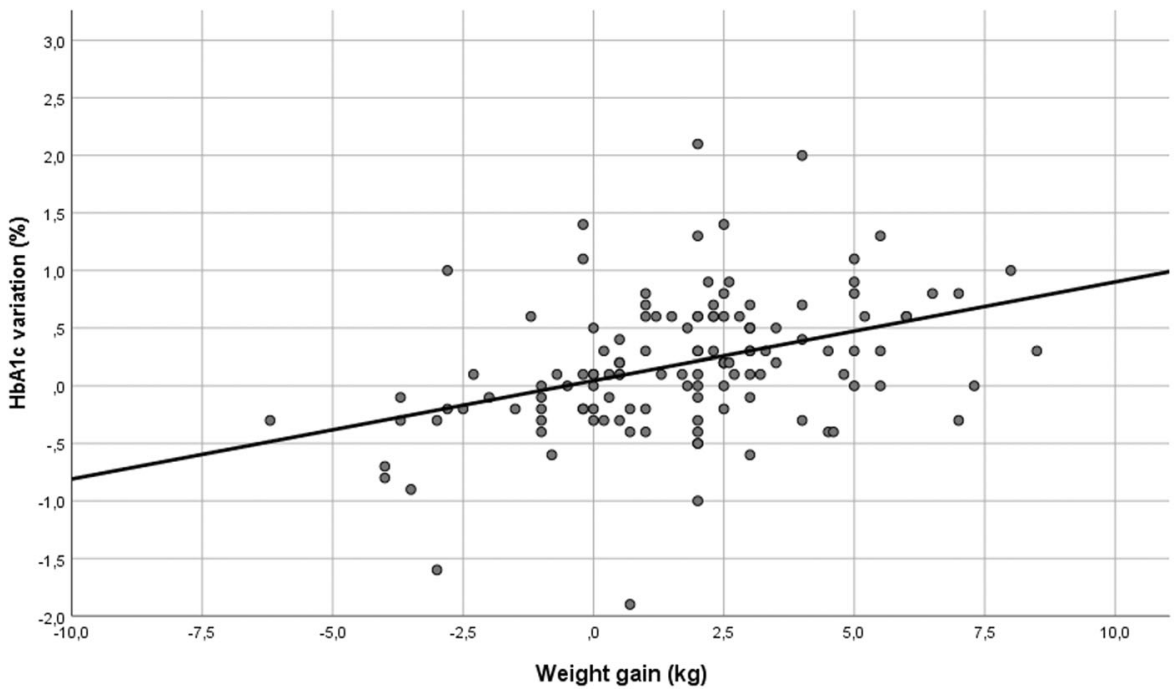

\begin{tabular}{lll}
\hline Variable & Univariate logistic regression & Multivariate logistic regression \\
& OR (C.I. $95 \% ; p$ value) & OR (C.I. 95\%; $p$ value) \\
\hline
\end{tabular}

\begin{tabular}{lc}
\hline Sex $(\mathrm{M})$ & $1.22(0.59-2.49 ; 0.588)$ \\
Age $>70$ years & $0.74(0.36-1.5 ; 0.407)$ \\
Disease duration (years) & $1(0.97-1.05 ; 0.744)$ \\
Weight $(\mathrm{kg})$ & $1.01(0.99-1.04 ; 0.115)$ \\
HbA1c $>7 \%$ & $0.61(0.3-1.25 ; 0.183)$ \\
Glycemia $(\mathrm{mg} / \mathrm{dl})$ & $0.997(0.985-1.009 ; 0.618)$ \\
Insulin therapy & $2.45(1.09-5.05 ; \mathbf{0 . 0 3 0})$ \\
SGLT2i & $1.75(0.75-4 ; 0.197)$ \\
GLP-1 RA & $0.83(0.39-1.75 ; 0.627)$ \\
DPP4i & $0.68(0.3-1.5 ; 0.350)$ \\
SU & $0.91(0.37-2.24 ; 0.840)$ \\
Pioglitazone & $1.21(0.38-3.86 ; 0.738)$ \\
Metformin & $0.7(1.28-1.8 ; 0.471)$ \\
Smoke & $0.90(0.42-1.93 ; 0.797)$ \\
Hypertension & $0.96(0.38-2-43 ; 0.939)$ \\
Dyslipidemia & $1.17((0.55-2.50 ; 0.679)$ \\
CAD & $2.43(0.99-5.96 ; \mathbf{0 . 0 5 2})$ \\
Heart Failure & $1.52(0.60-3.85 ; 0.374)$ \\
Stroke & $0.86(0.19-4.09 ; 0.867)$ \\
CKD (eGFR $\left.<60 \mathrm{~mL} / \mathrm{min} / 1.73 \mathrm{~m}^{2}\right)$ & $2.37(1.07-5.23 ; \mathbf{0 . 0 3 2})$ \\
Albuminuria $>20 \mathrm{mg} / \mathrm{l}$ & $1.65(0.62-4.34 ; 0.312)$ \\
Retinopathy & $0.57(0.24-1.33 ; 0.197)$ \\
Neuropathy & $1.69(0.56-5.15 ; 0.349)$ \\
\hline
\end{tabular}

$M$ male, $B M I$ body mass index, $W C$ waist circumference, $S G L T 2 i$ sodium glucose cotransporter 2 inhibitors, $G L P-1 \quad R A$ glucagon-like peptide-1 receptor agonists, DPP4i dipeptidyl peptidase IV inhibitors, $S U$ sulfonylureas, $C A D$ coronary artery disease, $C K D$ chronic kidney disease, N.A. not assessed.

Bold values indicates statistical significant $P<0.05$. control in T2DM patients is a risk factor for frequently unfavorable outcomes in SARS-CoV-2 infection [17-20].

Our study population was composed, on average, of obese diabetic patients with a well and stable glucose control. We found a significatively deterioration of all hallmarks of metabolic status (body weight, BMI, waist circumference, $\mathrm{HbA1c}$, and FPG) over time, compared with the pre-lockdown period. Based on the baseline BMI 
Table 4 Clinical and biochemical differences between insulin-treated patients and control group

\begin{tabular}{|c|c|c|c|}
\hline Baseline variable & $\begin{array}{l}\text { Insulin- } \\
\text { treated } \\
(N=42)\end{array}$ & $\begin{array}{l}\text { Not insulin- } \\
\text { treated } \\
(N=86)\end{array}$ & $P$ value \\
\hline Age (years) & $69.7 \pm 10.3$ & $69.9 \pm 9.1$ & 0.899 \\
\hline Disease duration (years) & $18.1 \pm 10$ & $14.1 \pm 8.2$ & 0.028 \\
\hline $\mathrm{HbA} 1 \mathrm{c} \%$ & $7.3 \pm 0.8$ & $6.9 \pm 0.8$ & 0.042 \\
\hline FPG (mg/dL) & $141.8 \pm 29.3$ & $136.6 \pm 29.4$ & 0.322 \\
\hline Weight (kg) & $80 \pm 21.8$ & $79.6 \pm 17.09$ & 0.926 \\
\hline BMI (kg/m2) & $30.3 \pm 7.1$ & $29.2 \pm 5.3$ & 0.387 \\
\hline WC $(\mathrm{cm})$ & $106 \pm 14.7$ & $102.6 \pm 12.05$ & 0.207 \\
\hline Hypertension & $36(85.7 \%)$ & $69(80.2 \%)$ & 0.448 \\
\hline Dyslipidemia & $32(76.2 \%)$ & $56(75.1 \%)$ & 0.204 \\
\hline Stroke & $3(7.1 \%)$ & $4(4.7 \%)$ & 0.560 \\
\hline CAD & $12(28.6 \%)$ & $20(23.3 \%)$ & 0.514 \\
\hline $\mathrm{HF}$ & $14(33.3 \%)$ & $11(12.8 \%)$ & 0.006 \\
\hline CKD & $20(47.6 \%)$ & $26(30.2 \%)$ & 0.054 \\
\hline $\begin{array}{l}\text { Microalbuminuria } \\
(>30 \mathrm{mg} / \mathrm{l})\end{array}$ & $11(26.2 \%)$ & $12(14 \%)$ & 0.090 \\
\hline Retinopathy & $17(40.5 \%)$ & $16(18.6 \%)$ & 0.008 \\
\hline Neuropathy & $8(19 \%)$ & $9(10.5 \%)$ & 0.179 \\
\hline Metformin & $27(64.3 \%)$ & $77(89.5 \%)$ & 0.001 \\
\hline SU & $6(14.3 \%)$ & $18(20.9 \%)$ & 0.366 \\
\hline DPP4-I & $5(11.9 \%)$ & $27(31.4 \%)$ & 0.017 \\
\hline SGLT2-I & $8(19 \%)$ & $25(29.1 \%)$ & 0.224 \\
\hline GLP-1 RA & $15(35.7 \%)$ & $27(31.4 \%)$ & 0.625 \\
\hline Pioglitazone & $1(2.4 \%)$ & $13(15.1 \%)$ & 0.030 \\
\hline
\end{tabular}

$F P G$ fasting plasma glucose, $B M I$ body mass index, $W C$ waist circumference, $C A D$ coronary artery disease, $H F$ heart failure, $C K D$ chronic kidney disease, $S U$ sulfonylureas, $D P P 4 i$ dipeptidyl peptidase IV inhibitors, $S G L T 2 i$ sodium glucose cotransporter 2 inhibitors, $G L P$ $1 R A$ glucagon-like peptide-1 receptor agonists.

Bold values indicates statistical significant $P<0.05$.

(i.e., $29.5 \mathrm{~kg} / \mathrm{m}^{2}$ ), one could argue that our population had a pre-existing poor lifestyle, predisposing to a further impairment of body composition during the lockdown period. Indeed, we can speculate that the home confinement during the COVID-19 pandemic had exacerbated all risk factors for weight gain, especially in overweight and obese T2DM patients, unfavorably modifying the eating and sleeping behaviors, as well as lifestyle [21]. This is consistent with other reports showing a worsening in dietary routine during the COVID-19 quarantine, especially in higher BMI individuals, regardless of diabetic disease $[4,5]$. In particular, a study on obese adults in Northern Italy showed an average weight increase of $1.5 \mathrm{~kg}$ after 1 month of lockdown, mainly associated with reduced exercise and increased intake of junk food [6]. Conversely, a study conducted in type 2 diabetic patients in South India found no significant change in body weight after quarantine.
This result probably arose because of the important differences in Indian lifestyle changes during the lockdown, since Indian patients increased vegetables and fresh food consumption and decreased fried and unhealthy snacks [11].

It is well established the impact of weight gain on increasing insulin resistance and worsening glucose metabolism in T2DM [7, 8]. Therefore, unsurprisingly, we have found that all the anthropometric indexes (body weight, BMI, and waist circumference) were directly associated with the glycated hemoglobin increase.

If we indeed consider type 1 diabetes mellitus, several recent studies on young and normal-weight patients using continuous glucose monitoring (CGM) systems found an unexpected improvement in glycemic control during the lockdown $[12,15,16]$. This can be explained by a more regular lifestyle during quarantine: the rhythm of daily activities slowed down, they were able to eat at more reproducible mealtimes, they were allowed to have a more controlled composition of meals [22]. Moreover, patients could spend more time on diabetes control, which is known to be a critical aspect of both insulin multi-injections or insulin pump therapy $[9,23]$. To date, scarce data are available on weight changes during the lockdown in T1DM patients [24].

To explain the different results obtained in T2DM patients, we should therefore consider that T1DM patients may have a higher awareness of the importance of a healthy lifestyle in disease management, and have a lower BMI at baseline $[8,9]$.

Regarding the anti-diabetic therapy, we indeed observed that insulin treatment was an independent predictor of metabolic control worsening, resulting in a more than doubled risk of $\mathrm{HbAlc}$ increasing. In patients affected by T2DM, insulin therapy is often used in long-term disease, or in presence of complications and other comorbidities, such i.e., the CKD or HF, which contraindicates other hypoglycemic drugs; moreover, it is usually associated with a reduced functional beta-cell activity. For this reason, endogenous insulin secretion is unable to cope with the increased insulin-resistance due to weight gain [7].

It is reasonable to suppose that T2DM patients are less confident in the self-optimizing of insulin dose in response to a worsened glycemic control. In fact, in contrast with T1DM patients, they are not used to handling the carbohydrate counting and the insulin correction factor; they rarely rely on technological devices such as flash glucose monitoring or continuous glucose monitoring (CGM) systems and they are often older [25, 26].

Another contributing negative factor may be the discouraged attendance to diabetes clinics or general practitioners due to the forced home staying. Moreover, it is reasonable to assume a more difficult retrieve of medical devices used to self-monitor blood glucose during the 
lockdown [3]. As a result, there was a limited chance to optimize insulin dose in response to an increase in sedentariness and worsening of dietary habits. Consequently, the lack of a proper insulin dose adjustment during quarantine may certainly have had a role in the worsened glycemic control in the lockdown period. This finding may therefore encourage the developing of structured educational program dedicated to T2DM patients.

Data regarding the actual contagious trend, unfortunately, prove that there might be other COVID-19 outbreaks in the next future, and, probably, shorter or longer periods of home confinement may be needed to contain the virus spread. Our study suggests that the best conditions to reach and maintain good metabolic control should be warranted to T2DM patients. Such strategies might include implementing telemedicine visits programs, offering a CGM system for self-glucose monitoring, and supplemental guidance encouraging diabetic patients to maintain a healthy lifestyle. This is especially valid in patients at higher risk of worsened glycemic control, like those undergoing insulin treatment.

Our results should be interpreted considering the intrinsic restrictions of a retrospective-observational study and the limited number of the sample size. Another limit of our study is the lack of detailed information on lifestyle changes in our population during the lockdown, like changes in dietary habits and exercise. We have hypothesized an increase in highly caloric food intake and diminished exercise in our sample, based on other surveys of similar populations and epidemiological and clinical characteristics of our patients at baseline. Because of this, it was not possible to recognize the specific impact of diet and physical activity during the lockdown on metabolic control. Therefore, it remains unclear whether the same results may be valid in younger and normal-weight DT2M subjects or patients using devices like the CGM sensors.

Despite the lack of precise data about changes in lifestyle during the lockdown, our strict inclusion criteria ensured that only a selected population with a stable weight and glycemic control, and without drug changes prior the lockdown was studied. On this basis we may infer that the increase in body weight and the consequently impairment of glycemic control are due to negative lifestyle modifications occurred during the lockdown period.

\section{Conclusions}

Our study highlights the negative impact of the lockdown effect on weight and glucose control in T2DM. Insulintreated patients were at higher risk to have a more difficult glucose control management and, for this reason, require more intensive medical attention.
Recognition of the lockdown effect is the first necessary step to take preventive measures to avoid the worsening of metabolic control in T2DM patients during an eventually needed period of home confinement.

Funding This research did not receive any specific grant from any funding agency in the public, commercial or not-for-profit sector.

\section{Compliance with ethical standards}

Conflict of interest The authors declare no competing interests.

Ethical approval The study was approved by the Ethical Committee of the Humanitas Research Hospital and a written consent was obtained by the enrolled subjects.

Publisher's note Springer Nature remains neutral with regard to jurisdictional claims in published maps and institutional affiliations.

\section{References}

1. L. Rosenbaum, The untold toll - the pandemic's effects on patients without Covid-19. N. Engl. J. Med. 382, 2368-2371 (2020). https://doi.org/10.1056/NEJMms2009984

2. S.R. Bornstein, F. Rubino, K. Khunti, G. Mingrone, D. Hopkins, A.L. Birkenfeld et al. Practical recommendations for the management of diabetes in patients with COVID-19. Lancet Diabetes Endocrinol. 8, 546-550 (2020). https://doi.org/10.1016/S22138587(20)30152-2

3. C. Koliaki, A. Tentolouris, I. Eleftheriadou, A. Melidonis, G. Dimitriadis, N. Tentolouris, Clinical management of diabetes mellitus in the era of COVID-19: practical issues, peculiarities and concerns. J. Clin. Med. 9, 2288 (2020). https://doi.org/10.3390/ jcm9072288

4. L. Di Renzo, P. Gualtieri, F. Pivari, L. Soldati, A. Attinà, G. Cinelli et al. Eating habits and lifestyle changes during COVID-19 lockdown: an Italian survey. J. Transl. Med. 18, 229 (2020). https://doi.org/10.1186/s12967-020-02399-5

5. A. Pietrobelli, L. Pecoraro, A. Ferruzzi, M. Heo, M. Faith, T. Zoller et al. Effects of COVID-19 lockdown on lifestyle behaviors in children with obesity living in Verona, Italy: a longitudinal study. Obesity 28, 1382-1385 (2020). https://doi.org/10.1002/ oby. 22861

6. M. Pellegrini, V. Ponzo, R. Rosato, E. Scumaci, I. Goitre, A. Benso et al. Changes in weight and nutritional habits in adults with obesity during the "lockdown" period caused by the COVID19 virus emergency. Nutrients 12, 2016 (2020). https://doi.org/10. 3390/nu12072016

7. M.J. Davies, D.A. D'Alessio, J. Fradkin, W.N. Kernan, C. Mathieu, G. Mingrone et al. Management of hyperglycemia in type 2 diabetes, 2018. A Consensus Report by the American Diabetes Association (ADA) and the European Association for the Study of Diabetes (EASD). Diabetes Care 41, 2669-2701 (2018). https://doi.org/10.2337/dci18-0033

8. American Diabetes Association, 5. Lifestyle management: standards of medical care in diabetes-2019. Diabetes Care 42, S46-S60 (2019). https://doi.org/10.2337/dc19-S005

9. A.D. Association, 1. Improving care and promoting health in populations: standards of medical care in diabetes - 2020 . Diabetes Care 43, S7-S13 (2020). https://doi.org/10.2337/ dc20-S001 
10. E. Fernández, A. Cortazar, V. Bellido, Impact of COVID-19 lockdown on glycemic control in patients with type 1 diabetes. Diabetes Res. Clin. Pr. 166, 108348 (2020). https://doi.org/10. 1016/j.diabres.2020.108348

11. P. Sankar, W.N. Ahmed, V. Mariam Koshy, R. Jacob, S. Sasidharan, Effects of COVID-19 lockdown on type 2 diabetes, lifestyle and psychosocial health: a hospital-based cross-sectional survey from South India. Diabetes Metab. Syndr. Clin. Res. Rev. 14, 1815-1819 (2020). https://doi.org/10.1016/j.dsx.2020.09.005

12. B. Capaldo, G. Annuzzi, A. Creanza, C. Giglio, R. De Angelis, R. Lupoli et al. Blood glucose control during lockdown for COVID19: CGM metrics in Italian adults with type 1 diabetes. Diabetes Care 43, e88-e89 (2020). https://doi.org/10.2337/dc20-1127

13. R.M. Anjana, R. Pradeepa, M. Deepa, S. Jebarani, U. Venkatesan, S.J. Parvathi et al. Acceptability and utilization of newer technologies and effects on glycemic control in type 2 diabetes: lessons learned from lockdown. Diabetes Technol. Ther. 22, 527-534 (2020). https://doi.org/10.1089/dia.2020.0240

14. A. Verma, R. Rajput, S. Verma, V.K.B. Balania, B. Jangra, Impact of lockdown in COVID 19 on glycemic control in patients with type 1 Diabetes Mellitus. Diabetes Metab. Syndr. Clin. Res. Rev. 14, 1213-1216 (2020). https://doi.org/10.1016/j.dsx.2020. 07.016

15. M. Longo, P. Caruso, M. Petrizzo, F. Castaldo, A. Sarnataro, M. Gicchino et al. Glycemic control in people with type 1 diabetes using a hybrid closed loop system and followed by telemedicine during the COVID-19 pandemic in Italy. Diabetes Res. Clin. Pr. 169, 108440 (2020). https://doi.org/10.1016/j.diabres.2020. 108440

16. Bonora B.M., Boscari F., Avogaro A., Bruttomesso D., Fadini G. P. Glycaemic control among people with type 1 diabetes during lockdown for the SARS-CoV-2 outbreak in Italy. 2020; 76949 Bytes. https://doi.org/10.6084/M9.FIGSHARE.12162024.V1

17. Mirani M., Favacchio G., Carrone F., Betella N., Biamonte E., Morenghi E., et al. Impact of comorbidities and glycemia at admission and dipeptidyl peptidase 4 inhibitors in patients with type 2 diabetes with COVID-19: a case series from an academic hospital in Lombardy, Italy. Diabetes Care. 2020. https://doi.org/ $10.2337 / \mathrm{dc} 20-1340$

18. A. Ceriello, E. Standl, D. Catrinoiu, B. Itzhak, N.M. Lalic, D. Rahelic et al. Issues of cardiovascular risk management in people with diabetes in the COVID-19 era. Diabetes Care 43, 1427-1432 (2020). https://doi.org/10.2337/dc20-0941

19. A. Kumar, A. Arora, P. Sharma, S.A. Anikhindi, N. Bansal, V. Singla et al. Is diabetes mellitus associated with mortality and severity of COVID-19? A meta-analysis. Diabetes Metab. Syndr. Clin. Res. Rev. 14, 535-545 (2020). https://doi.org/10.1016/j.dsx. 2020.04.044

20. I. Huang, M.A. Lim, R. Pranata, Diabetes mellitus is associated with increased mortality and severity of disease in COVID-19 pneumonia - a systematic review, meta-analysis, and metaregression. Diabetes Metab. Syndr. Clin. Res. Rev. 14, 395-403 (2020). https://doi.org/10.1016/j.dsx.2020.04.018

21. M.B. Ruiz-Roso, C. Knott-Torcal, D.C. Matilla-Escalante, A. Garcimartín, M.A. Sampedro-Nuñez, A. Dávalos et al. COVID-19 lockdown and changes of the dietary pattern and physical activity habits in a cohort of patients with type 2 diabetes mellitus. Nutrients 12, 2327 (2020). https://doi.org/10.3390/nu12082327

22. A.J. Ahola, S. Mutter, C. Forsblom, V. Harjutsalo, P.-H. Groop, Meal timing, meal frequency, and breakfast skipping in adult individuals with type 1 diabetes - associations with glycaemic control. Sci. Rep. 9, 20063 (2019). https://doi.org/10.1038/ s41598-019-56541-5

23. K. Rechenberg, R. Whittemore, M. Holland, M. Grey, General and diabetes-specific stress in adolescents with type 1 diabetes. Diabetes Res. Clin. Pr. 130, 1-8 (2017). https://doi.org/10.1016/j. diabres.2017.05.003

24. N. Shah, M. Karguppikar, S. Bhor, D. Ladkat, V. Khadilkar, A. Khadilkar, Impact of lockdown for COVID-19 pandemic in Indian children and youth with type 1 diabetes from different socioeconomic classes. J. Pediatr. Endocrinol. Metab. 34, 217-223 (2021). https://doi.org/10.1515/jpem-2020-0460

25. S.-Y. Chen, H.-C. Hsu, R.-H. Wang, Y.-J. Lee, C.-H. Hsieh, Glycemic control in insulin-treated patients with type 2 diabetes: empowerment perceptions and diabetes distress as important determinants. Biol. Res Nurs. 21, 182-189 (2019). https://doi.org/ 10.1177/1099800418820170

26. Zuo P., Shi J., Yan J., Yang L., Liu C., Yuan L., et al. Effects of insulin therapy and oral hypoglycemic agents on glycemic control for type 2 diabetes mellitus patients in china-a case control study. Exp Clin Endocrinol Diabetes. 2019; a-0881-9611. https://doi.org/ 10.1055/a-0881-9611 